\title{
Contexto e processo do Mapeamento Sistemático da Literatura no trajeto da Pós-Graduação no Brasil
}

\author{
Context and process of systematic \\ literature mapping in Brazilian \\ Graduate Education
}

\author{
Domício PROENÇA JÚNIOR' \\ Édison Renato SILVA²
}

\section{Resumo}

Este texto partilha elementos capazes de permitir a realização de um Mapeamento Sistemático da Literatura. O texto trata da natureza e contexto de um Mapeamento Sistemático da Literatura, enfatizando o significado específico do adjetivo "sistemático" como sendo a adoção de procedimentos para eliminar vieses (i) na localização de referências; (ii) na seleção das referências a serem consideradas; (iii) nos conteúdos das referências selecionadas; (iv) na extração de informações e entendimentos que as referências contêm; e (v) na apreciação crítica do conteúdo das referências. Discute ainda que a realização de um Mapeamento Sistemático seja responsabilidade de quem empreende uma Pós-Graduação e dá atenção ao contato inicial, panorâmico, com um determinado campo de conhecimento do qual um Mapeamento Sistemático depende. O texto também detalha cinco etapas do processo de realização de um Mapeamento Sistemático da Literatura, que correspondem (1) à busca por referências; (2) à coleta das referências para um repositório organizador; (3) à filtragem das referências; (4) ao relato dos resultados; (5) ao controle do processo, apresentando cada uma delas em termos gerais e detalhando em termos mais pragmáticos seu conteúdo. Considerações finais dão conta de algumas consequências da realização de Mapeamento Sistemático para a prática da pesquisa científica no Brasil.

Palavras-chave: Bases de dados referencias. Bibliografias sistemáticas. Pesquisa científica.

\begin{abstract}
We discuss the elements that support systematic literature mapping and the nature and context of systematic literature mapping, emphasizing the specific meaning of the adjective "systematic" as one that corresponds to the adoption of procedures that seek to eliminate biases (i) in the location of references; (ii) selection of significant references; (iii) content of selected references; (iv) extraction of information and understanding from references; and $(v)$ the critical appreciation of the content of references. We also argue that graduate students are responsible for their own systematic mapping and that systematic mapping depends on theattention paid to the panoramic, initial contact with a given field. We detail the five stages of systematic mapping process: (1) searching for references; (2) compiling references and organizing them into a system; (3) filtering/selecting references; (4) reporting the results; (5) controlling the process, presenting each stage in general terms before detailing more pragmatic aspects. Our final remarks address some of the consequences of producing systematic mapping for the practice of scientific research in Brazil.
\end{abstract}

Keywords: Reference databases. Systematic bibliographies. Scientific research.

\footnotetext{
1 Universidade Federal do Rio de Janeiro, Instituto Alberto Luiz Coimbra, Programa de Pós-Graduação em Engenharia de Produção. Rio de Janeiro, RJ, Brasil. 2 Universidade Federal do Rio de Janeiro, Escola Politécnica, Departamento de Engenharia Industrial. Caixa Postal 68507, 21941-972, Rio de Janeiro, RJ, Brasil. Correspondência para/Correspondence to: E.R. SILVA.E-mail:<edison@poli.ufrj.br>

Recebido em 24/7/2014, reapresentado em 17/6/2015 e aceito para publicação em 17/7/2015.
} 


\section{Introdução}

Um desafio àqueles que realizam o percurso da Pós-Graduação no Brasil é conseguir lidar de maneira adequada com o oceano de informações e possibilidades de fontes e referências franqueadas pela Web. Esse percurso contém em si mesmo um paradoxo distintivo que leva a problemas técnicos na busca, seleção, filtragem e composição de informações. O percurso da Pós-Graduação é, por um lado, uma jornada solitária: o transformar de si pela educação, em que o levantamento e apreciação das referências de maneira robusta e defensável perante os pares é uma tarefa largamente individual. Por outro lado, esse percurso é também uma vivência solidária, que aproveita o acervo do conhecimento e o diálogo vivo do momento dessa passagem, e pauta seu rumo e resultado pela meta de contribuir, de fazer parte de determinada comunidade concreta de estudiosos e praticantes. A solução desse paradoxo de maneira exitosa e satisfatória depende de um melhor conhecimento dos recursos informacionais disponíveis para o tratamento das informações, nessa jornada solitária percorrida com benefícios e relacionamentos de caráter solidário.

Este texto busca partilhar elementos capazes de fornecer soluções, alertar para a disponibilidade de recursos e auxiliar na realização da tarefa comum a qualquer projeto de pesquisa, independentemente de sua natureza: a construção do acervo de referências pertinentes a um determinado projeto de pesquisa (Borrego et al., 2014), num momento em que ainda não se pode contar, no curto prazo, com recursos de Web Semântica ou Web 2.0 (Ambinder \& Marcondes, 2013), dando a contrapartida de ambientação Whiteheadiana (Whitehead, 2008) à preocupação Deweyana do "letramento informacional" (Gasque \& Cunha, 2010), articulando-as em busca de uma compreensividade pragmática (Hendley, 1986).

O texto discute o que seja um Mapeamento Sistemático da Literatura, explicitando sua natureza (sistemático como eliminação de vieses) e contexto de aplicação. Em seguida, detalha seu processo em termos do arranjo de recursos informacionais disponíveis e discute algumas consequências de sua realização na prática da pesquisa científica no Brasil.
Ainda que orientada pela perspectiva de quem empreende a pesquisa associada a uma passagem pela Pós-Graduação, a exposição serve ainda a dois outros propósitos. Ela articula para docentes alguns dos recursos e possibilidades informacionais do presente. Apoia, ainda, o aperfeiçoamento das atividades de organização, documentação e oferta dos profissionais da Ciência da Informação, da qual dependem o vigor, o valor e a utilidade do acervo de informações. Nesse sentido mais amplo, este estudo pode contribuir para a discussão do lugar desses profissionais na produção e avanço do conhecimento científico.

\section{Natureza e contexto}

Estabelecer quais são as referências pertinentes para uma pesquisa depende de um levantamento da literatura de um campo científico. Esse levantamento se faz "sistemático" ao adotar heurísticas para a eliminação de vieses na consulta e uso de fontes. Um Mapeamento Sistemático é uma contribuição autoral que apresenta como resultado o estado da literatura de um determinado assunto ou subconjunto de assuntos, identificando as referências pertinentes a uma pesquisa. Por guiar os trabalhos que dele decorram, um Mapeamento Sistemático tem a carga de responsabilidade e compromisso ético de um trabalho profissional, respondendo à demanda por "integração", expressa em diversos campos científicos, inclusive as Ciências da Informação (Kelly \& Sugimoto, 2013).

Diz-se "sistemático", em emulação às práticas de Evidence-Based practices, especialmente da Medicina, por adotar procedimentos que buscam eliminar vieses (i) na localização de referências; (ii) na seleção das referências a serem consideradas; (iii) nos conteúdos das referências selecionadas; (iv) na extração de informações e entendimentos que as referências contêm e (v) na apreciação crítica do conteúdo das referências (Khan et al., 2001).

A meta de um Mapeamento Sistemático é relatar o que encontra de pertinente para uma dada pesquisa (ou, mais amplamente, para um determinado assunto) em todos os espaços e atividades que conformam o assunto até o momento de sua realização. Considera o que se pode encontrar em fontes indexadas (as disponíveis no Portal de Periódicos da Coordenação de Aper- 
feiçoamento de Pessoal de Nível Superior [Capes]) ou acessíveis a partir de bancos de dados (o catálogo da Biblioteca do Congresso dos Estados Unidos da America) e serviços de bibliotecas (do Programa de Comutação Bibliográfica [Comut]). Considera também as diversas formas ditas "literatura cinza" (que variam de campo para campo, por exemplo, artigos e contribuições em eventos, relatórios técnicos ou posts em blogs), cuja incorporação e apreciação ao acervo de conhecimento vêm sendo cada vez mais reconhecidas (Arboit \& Bufrem, 2011; Pejsova \& Vaska, 2011; Lawrence, 2012). Considera ainda como fontes grupos (associações, institutos profissionais ou grupos universitários de pesquisa) ou produtos escolares (dissertações e teses) que contribuem para o assunto.

Existem diversas formas de lidar com essa problemática, que admite um olhar integrativo entre busca e aproveitamento sob restrições (Bates, 2002), com diferentes abordagens e resoluções (Graziotin et al., 2014; Michels \& Fu, 2014). Uma das mais ambiciosas é a que considera a tarefa da reunião de referências como análoga à caça e à coleta, isto é, uma ronda de busca guiada pelo "princípio do menor esforço", e portanto sujeita às diversas idiossincrasias e desdobramentos particulares de trajetos otimizantes de esforço (Pirolli, 2009). Seguir o princípio do menor esforço corresponde a um viés indefensável no contexto específico do trajeto de Pós-Graduação stricto sensu, vulnerável a fenômenos como o viés de distribuição ordinal - power law de citações (Brzezinski, 2015). A questão é dupla. Primeiro, a qualidade do percurso formativo que edifica uma medida de erudição (especialmente no doutoramento). Segundo, a necessidade de dar conta, de maneira defensável, do acervo do todo que seja relevante para a pesquisa. Isso condena a reificação de vieses segundo o princípio do menor esforço como sendo"naturais" (Bigdeli et al., 2013, p.108). A descrição, mesmo que matizada, do que se observa serem práticas também não é suficiente, não importa o quão atentas ou detalhadas (Palmer et al., 2009). O que se propõe aqui é bastante diverso. Trata-se de propor um tipo de "princípio do esforço suficiente" para se chegar a um resultado academicamente defensável, isto é, que seja capaz de explicar documentadamente os limites e os critérios de sua busca, de sua coleta e da seleção de referências a serem trabalhadas.
A realização de um Mapeamento Sistemático é contextualizada pela especificidade da dinâmica do assunto e pela adoção de heurísticas. $\mathrm{O}$ assunto pode ter a sua dinâmica dominante em um ou em outro veículo ou espaço. Há assuntos em que livros são mais importantes que artigos, por exemplo. Entretanto, sempre se pode buscar identificar autores ou instituições cujo uso ou referenciação edificam os termos de relacionamento de uma comunidade de estudiosos e praticantes, complementando o processo de mapeamento do assunto. Quando se avança para além do que a busca tópica pode produzir nas fontes de referências, trata-se de considerar heurísticas como a do uso de autores ou obras como sementes de busca de referências. Trata-se propriamente de heurísticas porque não se pode garantir que tais procedimentos produzam o resultado desejado.

O processo de identificação de fontes pertinentes ao projeto de pesquisa é recursivo. A partir de uma primeira busca, por exemplo, por palavras-chave, pode-se chegar a autores ou obras diretamente pertinentes para a questão de pesquisa. Tais autores ou obras podem servir como sementes para novas buscas. Assim, por exemplo, podem-se mapear os relacionamentos entre referências pelas obras que citam ou pelas quais são citadas. É necessário ter documentadamente critérios pelos quais adjudicar a pertinência de uma dada referência. Esse não é um procedimento automático e cego, mas uma tomada de decisão substantivamente defensável sobre quais trabalhos incluir ou excluir. O entrecruzamento de referências citadas e citantes, com um critério de pertinência à questão no assunto, identifica a tessitura dos relacionamentos de uma comunidade.

Note-se que pode haver uma referência pertinente segundo os critérios adotados que não cita nem é citada por nenhuma outra referência. Ainda por isso, trata-se de heurísticas, que auxiliam o tratamento das fontes de referências para além do que é possível fazer através das tradicionais buscas por palavras-chave. Na realidade brasileira, parece bastante provável que a realização de um Mapeamento Sistemático seja responsabilidade de quem empreende uma Pós-Graduação. Daí, a necessidade de associar o retorno de fontes de referências com a adoção de heurísticas auxiliares defensáveis de maneira documentada (Hart, 2009). 
A construção de um panorama suficiente para o início de um Mapeamento Sistemático beneficia-se de uma leitura preparatória, a ser conduzida de forma mais ou menos canônica (Adler \& van Doren, 2011). Usualmente, é suficiente que se dê conta de uma escolha defensável do seguinte conjunto de obras, tipicamente livros: a Introdução do Assunto; a História do Assunto; os Clássicos do Assunto; as Questões Contemporâneas do Assunto; o Futuro do Assunto; o Handbook do Assunto (que agremia as informações e dados para aplicação, um vade mecum) - a que se pode ter a fortuna de poder somar, em alguns assuntos, o Sourcebook do Assunto (que resume o resultado e indica o conjunto das fontes, usualmente as fontes primárias, de dados, teorias, escolas, abordagens, eventos e personalidades) ou o Databook do Assunto (que disponibiliza de maneira agregada e consistente o conjunto dos dados empíricos). Cada vez menos frequentemente em mídia impressa, por razões evidentes, deve-se encontrar A Bibliografia do Assunto. Estes " $n$ "livros são o melhor roteiro e a primeira leitura para se aproximar de um determinado assunto. Pode haver assuntos nos quais se tem um ou mais veículos (periódicos, sites, ou eventos) que podem servir para tal contato; mas mesmo então sua propriedade é medida pelo quanto se aproximam do equivalente a esses livros. A questão é estruturar o que sejam o objeto de estudo, seu propósito e destinação o"XYZ" de Booth et al. (2008).

\section{Processo}

O processo de mapeamento sistemático de literatura aqui proposto envolve cinco etapas aplicadas recursivamente a partir da ambientação obtida pela leitura dos" "n"livros: (1) a busca por referências; (2) a coleta das referências para um repositório organizador; (3) a filtragem das referências; (4) o relato dos resultados; (5) o controle do processo, em paralelo com as etapas anteriores e documenta o trajeto de realização do Mapeamento Sistemático (Figura 1).

1) A busca por referências decorre da consulta a determinadas fontes, indexadas ou não. Em larga medida, a escolha de determinadas fontes e das formas pelas quais consultá-las já expressa um alinhamento com o que sejam as prioridades e práticas de determinada comunidade de estudiosos e praticantes (Maxwell, 2013). No caso brasileiro, a disponibilidade do acervo dos pe-

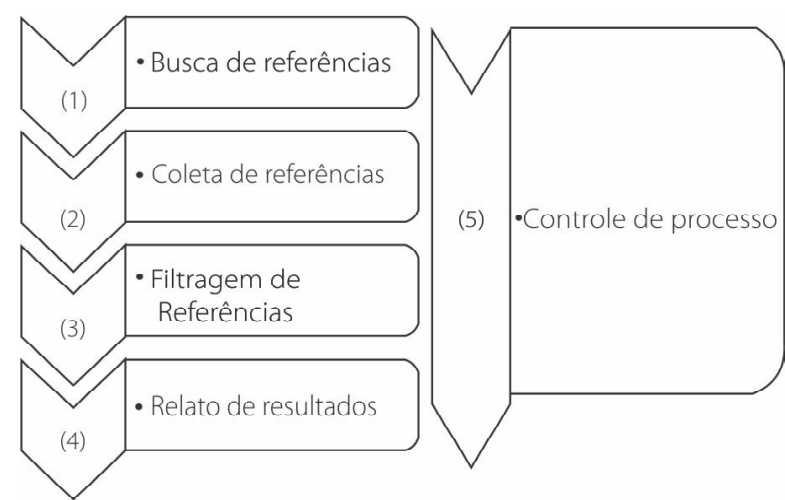

Figura 1. Processo de mapeamento sistemático. Fonte: Elaborada pelos autores (2015).

riódicos CAPES e as regras da avaliação trienal da Pós-Graduação emprestam uma medida substancial de homogeneidade para algumas dessas fontes e formas de consulta. Assim, por exemplo, tanto o acesso ao Web of Science quanto a consulta recursiva e documentada por suas ferramentas de busca é algo que todos os trajetos de Pós-Graduação brasileiros deveriam ter em comum. De maneira mais branda, mas não menos significativa, o mesmo se poderia dizer do acesso à Biblioteca Digital de Teses e Dissertações do Instituto Brasileiro de Informações em Ciência e Tecnologia e seu equivalente internacional no Proquest. Daí em diante, porém, tem-se a particularidade de fontes e formas de acesso que conformam o acervo de saberes de uma dada comunidade, para a qualidade do qual os esforços e talentos de profissionais da informação se apresentam como um fator de delicada e importante relevância (Rodrigues \& Fachin, 2010).

A qualidade dos resultados da busca por palavras-chave depende da maneira pela qual se organiza a informação nas fontes de referências. No caso de fontes estruturadas por tesauro, como o PubMed/MedLine, a busca direta por termos indexados permite que se obtenha uma fração considerável do acervo do conhecimento para um projeto de pesquisa de maneira defensável e documentada (Richter \& Austin, 2012). Entretanto, para quase todos os outros campos de conhecimento, não existem ainda bases que possuam tesauro e que sejam continuamente atualizadas para garantir a indexação das 
referências nos termos de um tesauro (Lipscomb, 2000). Nesses casos, só o uso de heurísticas permite identificar referências com resultados que se aproximem aos de uma busca apoiada em tesauro. Essas heurísticas dependem da exploração das relações entre referências (referências citadas e citantes), das autorias e coautorias, dos eventos, bem como dos veículos e instâncias em que foram publicadas. Pela aplicação recursiva e documentada desse conjunto de heurísticas de buscas, é possível que um projeto de pesquisa chegue a resultados defensáveis de qualidade comparável a buscas em fontes indexadas por tesauro.

2) A coleta das referências para um repositório organizador se traduz inescapavelmente na escolha e fluência do uso de determinados softwares (Lorenzetti \& Ghali, 2013). Aqui se apresentam diferentes escolhas em termos de hardware e de opções de linhagens de software ao redor de um determinado software bibliográfico e dos diversos outros softwares de apoio, cujo conjunto corresponde a um determinado workflow acadêmico. Isso corresponde a um projeto customizado e idiossincrático do sistema de gestão de informação bibliográfica, capaz de registrar documentadamente o resultado das consultas e decisões na realização do Mapeamento Sistemático. Tem-se diversidade de interfaces e possibilidades de captura e tratamento da informação nas diferentes versões dos softwares (Cordon-Garcia et al., 2009). Quaisquer que sejam os arranjos específicos que se adotem, eles devem ser capazes de economizar tempo na coleta e filtragem de referências, por um lado, e apoiar a busca dos fins do projeto de pesquisa, por outro.

A economia de tempo depende fundamentalmente da customização de interfaces humano-máquina e software-software - por exemplo, em termos básicos, acertar as configurações que permitem nomear, classificar, salvar e documentar referências e arquivos digitais na forma mais afeita ao usuário de maneira automática. Em termos mais avançados, trata-se de adotar os scripts que permitem o trânsito do conteúdo de referências selecionadas e dos comentários de estudo, para dar-lhes tratamento cognitivo.

O apoio aos fins da pesquisa depende de um trabalho autoral de construção de categorias, classes, agendas de trabalho, resumos e conexões difusas. Em termos básicos, isso diz respeito à estrutura de diretórios e pastas de diversos tipos que articulam o mesmo acervo de referências e arquivos de diferentes maneiras. Isso permite a diversidade de formas de recuperação da informação e perspectivas do entendimento das quais o lampejo do entendimento ou a fagulha da criatividade podem depender. Em termos mais avançados, isso diz respeito aos sistemas de classificação capazes de expressar dife-rentes dimensões de cognição do tratamento de infor-mações e articulação de entendimentos, edificando a capacitação de quem realiza a pesquisa. Em ambos os casos, o que se tem como pauta é a adaptação das ferramentas e procedimentos ao alcance e aos limites da cognição humana: seja ganhando tempo e evitando erros, seja preservando resultados e conteúdos autorais para além do que se poderia lembrar.

3) A filtragem das referências corresponde ao exercício do juízo e à articulação de um entendimento em construção, que materializa a seleção e uso dos conteúdos das referências. Trata-se de ter interfaces e arranjos de software afeitos, ou mesmo idênticos, à maneira como se pensa e se vai entendendo, ao longo do tempo, um determinado assunto. Isso admite diversas passagens, idas e vindas, cuja documentação automática faz menos demandantes. Mas, essencialmente, trata-se do exercício da cognição e entendimento de quem empreende a pesquisa, recorrentemente selecionando quais referências ler, de que maneira, para que fins, e ajuizando quando ampliar, restringir ou encerrar o processamento de mais referências.

Em termos pragmáticos, isso corresponde a passos sucessivos de seleção e exclusão de referências em um workflowacadêmico apoiado por um sistema de gestão de informação bibliográfica. Isso permite registrar, consolidar, retornar e modificar as decisões tomadas dentro de uma determinada estrutura de projeto de pesquisa, a "ampulheta" de (Maxwell, 2013, p.5). O que torna um Mapeamento de Literatura sistemático é a capacidade de documentar critérios de filtragem e estágios de inspeção das referências consideradas e ajuizadas em cada um deles. Os critérios de filtragem quanto à pertinência de referências para um projeto de pesquisa são autorais e portanto, em última análise, enviesados. O que diferencia um mapeamento sistemático é a explicitação documentada dos critérios utilizados, dos mecanismos de sua aplicação e das referências identificadas pela 
busca mas excluídas como resultado da aplicação dos critérios. Em termos básicos, a aplicação dos critérios de filtragem se dá por estágios sucessivos, sendo o primeiro deles a consideração das palavras-chave, título e abstract. No segundo estágio, é necessário ter o texto completo, cuja leitura inspecional do conteúdo e bibliografia apoia a aplicação dos critérios. Daqui em diante, tem-se diversidade metodológica entre campos e mesmo entre diferentes projetos de pesquisa: admitem-se diferentes formas de apreciação do conteúdo para produzir sua classificação no Mapeamento Sistemático.

4) O relato dos resultados, ou seja, a comunicação do Mapeamento Sistemático da Literatura, admite variedade. No caso, como o foco deste texto é a realização individual de um mapeamento como parte de pesquisa de Pós-Graduação, não se têm requisitos de validação ou acreditação dos resultados por outros fora das Ciências Médicas. No entanto, em todas as disciplinas científicas, o resultado de seu uso é objeto de avaliação pelos pares, na defesa de tese ou dissertação. Tudo o que é necessário é a verificação documentada de que os resultados correspondem, de fato, à aplicação das heurísticas, de maneira a ambicionar a eliminação dos vieses (i) na localização de referências; (ii) na seleção das referências a serem consideradas; (iii) nos conteúdos das referências selecionadas; (iv) na extração de informações e entendimentos que as referências contêm e (v) na apreciação crítica do conteúdo das referências, de maneira a compor o que seja o estado da literatura de uma determinada comunidade. Em termos pragmáticos, isso corresponde à comunicação autoral de quais sejam, para o campo mapeado, os principais artigos, periódicos científicos, congressos, livros e séries de livros, teses e dissertações, autores, fontes de literatura cinza, grupos de pesquisa e associações profissionais, de forma compatível com os padrões de bibliotecas digitais (Schmalbeck et al., 2006).

5) O controle do processo seria, idealmente, um resultado automático do uso dos softwares. Essa realidade ainda está muito distante, precisamente porque diferentes pesquisadores, em diferentes campos, diante de diferentes questões de pesquisa, dependem da idiossincrasia de seu entendimento e percurso para poderem avançar o conhecimento. Mesmo diante do PubMed/MedLine, as decisões que não são do ou no software necessitam de registro e acompanhamento pelos limites da cognição e memória humanas. Isso significa, inescapavelmente, que é necessário construir e utilizar um sistema de controle (que pode ser ele mesmo o objeto do uso de um determinado software) capaz de documentar de maneira completa o processo de realização de um Mapeamento Sistemático em cada um de seus estágios, coligindo e permitindo articular a diferente etapa de sua implementação e mesmo, talvez principalmente, as mudanças no entendimento de quem o realiza por que o realiza.

Em termos pragmáticos, isso corresponde ao controle do acesso às diferentes fontes de referências e ao que sejam as heurísticas, os critérios e o resultado das filtragens para cada uma das diferentes fontes e diante de cada uma das diferentes classificações que vão sendo construídas ao longo do Mapeamento. Em termos básicos, pode-se configurar uma planilha eletrônica para registrar os passos do workflow acadêmico. Em termos avançados, um determinado projeto pode emular a estrutura de acompanhamento de projetos, beneficiando-se de ferramentas mais robustas (Cooper, 1990). Novamente, a questão é como permitir a ampliação da cognição e memória humana para dar conta da amplitude e da repetição recursiva de procedimentos semelhantes em diferentes momentos e com diferentes prioridades, mas diante da mesma tela de computador.

\section{Conclusão}

Este texto buscou partilhar elementos capazes de permitir a realização de um Mapeamento Sistemático da Literatura, no percurso da Pós-Graduação no Brasil.

Inicialmente, o texto tratou da natureza e contexto de um Mapeamento Sistemático da Literatura, enfatizando três aspectos. O primeiro é o significado específico do adjetivo "sistemático" como correspondendo à adoção de procedimentos de natureza heurística capazes de eliminar vieses (i) na localização de referências; (ii) na seleção das referências a serem consideradas; (iii) nos conteúdos das referências selecionadas; (iv) na extração de informações e entendimentos que as referências contêm; e (v) na apreciação crítica do conteúdo das referências. O segundo é a constatação de que, diante da realidade brasileira, parece bastante provável que a realização de um Mapeamento Sistemático seja responsabilidade de quem empreende uma Pós-Graduação, sendo oportuno apresentar o caráter recursivo da aplicação de heurísticas. O terceiro é a atenção para 
com o contato inicial, panorâmico, com um determinado campo de conhecimento, a partir do qual é possível iniciar o empreendimento de um Mapeamento Sistemático. Isso correspondeu à caracterização dos " $n$ " livros que permitem realizar um primeiro contato suficientemente amplo mas manejável.

O texto deu conta, em algum detalhe, do processo de realização de um Mapeamento Sistemático da Literatura. Apresentou as cinco etapas que, aplicadas recursivamente a partir da ambientação obtida pela leitura dos " $n$ "livros, correspondem (1) à busca por referências; (2) à coleta das referências para um repositório organizador; (3) à filtragem das referências; (4) ao relato dos resultados; (5) ao controle do processo, apresentando cada uma delas em termos gerais e detalhando em termos mais pragmáticos seu conteúdo.

O contexto de realização individual de um Mapeamento Sistemático da Literatura não é despido de consequências. É forçoso reconhecer que, em tal circunstância, o trabalho de fazer o resultado de um Mapeamento plenamente inteligível a qualquer outro não pertence intrinsecamente à sua realização no contexto de um percurso de Pós-Graduação. No entanto, esse trabalho adicional de comunicação do Mapeamento pode fazer parte das prioridades e práticas da comunidade de estudiosos e praticantes em que uma determinada pesquisa se insere, num determinado momento e circunstâncias de trabalho (em contraste com a perspectiva passada da pesquisa em papel e futura, da Web 2.0). Isso não é uma escusa para que se tenha um Mapeamento mal-ajambrado no presente e no curto prazo, porque se adotam heurísticas e se depende de sua cumulação ajuizada para aproximar um determinado resultado sistemático. Ao contrário, precisamente porque a pesquisa de quem faz um Mapeamento depende de sua qualidade, tem-se toda a motivação para que ele seja o melhor ao alcance das fontes, heurísticas e recursos. A questão pragmática é bem mais simples: o Mapeamento pode permanecer nos softwares em que foi produzido, sem ser formatado nem decodificado para além daquilo considerado suficiente por quem o fez e o usa. Entretanto, qualquer que seja o tipo de pesquisa realizada, seja um trabalho de natureza eminentemente teórica, seja um trabalho de natureza eminentemente prática, a inserção da pesquisa numa comunidade de estudiosos e praticantes depende da identificação da pertinência da contribuição ao avanço do conhecimento científico, que só é possível com recurso a um Mapeamento Sistemático da Literatura.

A questão do controle do processo, a seu turno, tem uma segunda face, que espelha o paradoxo solitário e solidário apontado no início deste artigo. À face solitária da passagem pela Pós-Graduação corresponde o registro do próprio trajeto de entendimento e capacitação de quem o realiza - passagem essa em que a realização de um Mapeamento Sistemático da Literatura tem um papel de ampliação de horizontes e alargamento de entendimento, educando o juízo. Tem-se, conclusivamente, de outra maneira, a mesma questão da humanidade de quem realiza a pesquisa que se buscou tratar quando se deu atenção a um processo apoiado em softwares num mundo digital. Há exemplos conhecidos do alcance e importância desse tipo de artesanato intelectual. Têm-se os arquivos de Thomas Alva Edison, com os originais integrais agrupados em pastas autorais com o mais mínimo de comentário, sua estrutura sendo o elemento autoral. Tem-se a Zettelkasten de Niklas Luhmann, em que a estrutura do acervo do saber foi reconstruída em moldes e códigos próprios, constituindo o processo mesmo de seu mapeamento e apreciação. Mais modestamente, e mais ao alcance de quem empreende a Pós-Graduação, têm-se as considerações de Charles Wright-Mills sobre um diário de pesquisa. Talvez em termos mais amplos e mais adequados, tem-se ainda a pertinência de um commonplace book, onde quem pesquisa e empreende um Mapeamento Sistemático coloca - e pode encontrar de si para si -, suas dúvidas, ideias, devaneios, escolhas, decisões, estudos, rascunhos, problemas, soluções, registrando seu percurso no conhecer-se a si mesmo ao aprender.

\section{Agradecimentos}

Agradecem aos comentários e sugestões dos revisores anônimos pelo processo de revisão e ao Conselho Nacional de Desenvolvimento Científico e Tecnológico como a concessão da bolsa de produtividade de pesquisa, PQ-II. Processo n 307185/2014-1.

\section{Colaboradores}

Todos os autores contribuíram na concepção e desenho do estudo, análise de dados e redação final. 


\section{Referências}

Adler, M.; Van Doren, C. Como ler livros. Rio de Janeiro: É Realizações, 2011.

Ambinder, D.M.; Marcondes, C.H. New experiences for presenting, accessing, and reading digital scientific articles on the Web. Transinformação, v.25, n.3, p.195-201, 2013.

Arboit, A.E.; Bufrem, L.S. The production of scientific research in national events in the field of information science. Transinformação, v.23, n.3, p.207-217, 2011.

Bates, M.J. Toward an integrated model of information seeking and searching. New Review of Information Behaviour Research, n.3, p.1-15, 2002.

Bigdeli, Z. et al. Patterns of authors' information scattering: Towards a causal explanation of information scattering from a scholarly information-seeking behavior perspective. Scientometrics, v.96, n.1, p.103-131, 2013.

Booth, W.C.; Colomb, G.G.; Williams, J.M. The craft of research. Chicago: University of Chicago Press, 2008.

Borrego, M.; Foster, M.J.; Froyd, J.E. Systematic literature reviews in engineering education and other developing interdisciplinary fields. Journal of Engineering Education, v.103, n.1, p.45-76, 2014.

Brzezinski, M. Power laws in citation distributions: Evidence from Scopus. Scientometrics, v.103, n.1, p.213-228, 2015.

Cooper, R.G. Stage-gate systems: A new tool for managing new products. Business Horizons, v.33, n.3, p.44-54, 1990.

Cordon-Garcia, J.A.; Martin-Rodero, H.; Alonso-Arevalo, J. Generation reference management software: Comparative analysis of ReWorks, EndNote web and Zotero. Profesional de la Informacion, v.18, n.4, p.445-454, 2009

Gasque, K.C.G.D.; Cunha, M.V.The John Dewey's epistemology and informational literacy. Transinformação, v.22, n.2, p.139-146, 2010.

Graziotin, D.; Wang, X.; Abrahamsson, P. A framework for systematic analysis of open access journals and its application in software engineering and information systems. Scientometrics, v.101, n.3, p.1627-1656, 2014.

Hart, C. Doing a literature review. Thousand Oaks: Sage, 2009.

Hendley, B.P.A.N. Dewey, Russell, Whitehead philosophers as educators. Carbondale: Southern Illinois University Press, 1986.

Kelly, D.; Sugimoto, C.R. A systematic review of interactive information retrieval evaluation studies, 1967-2006. Journal of The American Society for Information Science and Technology, v.64, n.4, p.745-770, 2013.
Khan, K.S. et al. Undertaking systematic reviews of research on effectiveness: CRD's guidance for carrying out or commissioning reviews. York: University of York, 2001. Available from:<http://eprints.whiterose.ac.uk/1139/>.Cited: Jul. 16, 2015.

Lawrence, A. Electronic documents in a print world: Grey Literature and the Internet. Media International Australia, n.143, p.122-131, 2012.

Lipscomb, C.E. Medical subject headings (MeSH). Bulletin of the Medical Library Association, v.88, n.3, p.265-266, 2000.

Lorenzetti, D.L.; Ghali, W.A. Reference management software for systematic reviews and meta-analyses: An exploration of usage and usability. BMC Medical Research Methodology, v.13, n.141, 2013. doi: 10.1186/1471-2288-13-141.

Maxwell, J.A. Qualitative research design: An interactive approach. Thousand Oaks: Sage Publications, 2013.

Michels, C.; Fu, J. Systematic analysis of coverage and usage of conference proceedings in web of Science. Scientometrics, v.100, n.2, p.307-327, 2014

Pejsova, P.; Vaska, M. An analysis of current grey literature document typology. Prague: Greynet, 2011. (GL Conference Series).

Palmer, C.L., Teffeau, L.C.; Pirmann, C.M. Scholarly information practices in the online environment:Themes from the literature and implications for library service development. Report Commissioned by OCLC Research. 2009. Available from: <http// www. oclc.org/programs/publications/reports/2009-02.pdf>. Cited: Jul. 16, 2015.

Pirolli, P. Information foraging theory: Adaptive interaction with information. New York: Oxford University Press, 2009.

Richter, R.R.; Austin, T.M. Using MeSH (Medical Subject Headings) to enhance PubMed search strategies for evidencebased practice in physical therapy. Physical Therapy, v.92, n.1, p.124-132, 2012

Rodrigues, R.S.; Fachin, G.R.B. Portal of scientific journals: A multidisciplinary work. Transinformação, v.22, n.1, p.33-45, 2010.

Schmalbeck, L.; Stuart-Moore, J.; Evans, M. Adapting peer verification, validation and accreditation processes for digital libraries. New York: Association of Computing Machinery, 2006.

Whitehead, A.N. The aims of education and other essays. Princeton: Recording, 2008. 